\title{
Alterações hematológicas em pacientes infectados pelo vírus da imunodeficência humana submetidos à terapia antirretroviral com e sem inibidor de protease Hematological abnormalities in patients infected with human immunodeficiency virus on antiretroviral therapy with and without protease inhibitors
}

Elaine N. Daminelli ${ }^{l}$

Arício Tritinger ${ }^{2}$

Celso Spada

\begin{abstract}
A anemia é uma anormalidade hematológica comumente encontrada em pacientes infectados pelo vírus da imunodeficiência humana (HIV) e sua prevalência estimada entre 63\% a 95\%. O objetivo deste estudo foi avaliar as alterações hematológicas e o Índice de Massa Corporal (IMC) de pacientes HIV soropositivos com ou sem uso de terapia antirretroviral com e sem inibidor de protease. Os pacientes HIV soropositivos foram diagnosticados pelo teste de enzimaimunoensaio (ELISA) e confirmados por imunofluorescência. As alterações hematológicas foram determinadas por aparelho de automação Coulter Maxim Autoloader ${ }^{\circledR}$, contagem de células $\mathrm{CD} 4+$ e $\mathrm{CD} 8+$ por citometria de fluxo FACSCount $\AA^{\circledR}$ e carga viral por amplificação baseada na sequência do ácido nucleico - Nucleic Acid Sequence Based Amplification (NASBA®). A avaliação dos dados hematológicos demonstrou níveis diminuídos no número de leucócitos e hemoglobina no grupo de estudo que fazia uso de terapia antirretrovial, quando comparado ao grupo controle sem uso desta terapia; resultado semelhante verificouse também para o IMC dos pacientes HIV soropositivos $(p<0,0001, p=0,006$ e $p<0,0001)$ respectivamente. Não houve diferença significativa entre os grupos que faziam uso de terapia antirretrovial com e sem inibidores de protease (IP). A avaliação dos dados hematológicos associada à contagem de células CD4+ e quantificação da carga viral pode contribuir para o monitoramento da infecção e auxiliar na tomada de decisão a respeito da intervenção clínica mais adequada nestes pacientes. Rev. Bras. Hematol. Hemoter.
\end{abstract}

Palavras-chave: Anemia; HIV/AIDS; tratamento combinado; inibidores de trascriptase reversa.

\section{Introdução}

A infecção pelo vírus da imunodeficiência adquirida (HIV) é caracterizada por uma contínua replicação viral e depleção dos linfócitos T CD4+, acarretando alterações imunológicas e infecções por patógenos oportunistas. ${ }^{1} \mathrm{~A}$ carga viral e a contagem de células T CD4+ são marcadores prognósticos importantes para o monitoramento da infecção pelo HIV e acompanhamento da evolução da doença nesses pacientes. Outros fatores, como infecções oportunistas, marcadores de ativação imunológica e perda de peso dos pacientes, também estão associados com a progressão da doença causada pelo HIV. Em alguns casos, estes fatores, podem estar relacionados com a diminuição da sobrevida, os

${ }^{I}$ Farmacêutica/Bioquímica.

${ }_{3}^{2}$ Professor de Bioquímica Clínica do Depto. de Análises Clínicas da Universidade Federal de Santa Catarina/UFSC - Florianópolis-SC.

${ }_{3}^{3}$ Professor de Hematologia Clínica do Depto. de Análises Clínicas da Universidade Federal de Santa Catarina/UFSC - Florianópolis-SC.

Universidade Federal de Santa Catarina/UFSC - Florianópolis-SC.

Correspondência: Celso Spada

Universidade Federal de Santa Catarina

Centro de Ciências da Saúde - Departamento de Análises Clínicas - Campus Universitário - Trindade

Caixa Postal 476

88040-900 - Florianópolis-SC - Brasil

Tel.: (55 48) 3721-9712 Ramal 222; (55 48) 9973-2656; Fax: (55 48) 3721-9542

E-mail:celso@ccs.ufsc.br 
quais podem ser relacionados entre os indicadores da gravidade e/ou progressão da doença. ${ }^{2,3}$

Estudos epidemiológicos recentes têm relatado que a anemia é uma das manifestações hematológicas mais comuns na infecção pelo HIV, com prevalência entre $63 \%$ a $95 \%$ dependendo do estado clínico do paciente. ${ }^{4}$ A etiologia da anemia em pacientes infectados pelo HIV é de natureza multifatorial, podendo estar relacionada a infecções oportunistas, deficiências nutricionais (ferro, vitamina B12 e ácido fólico), determinadas medicações (antibióticos e agentes antirretrovirais) e doenças invasivas na medula óssea que provocam alterações nas células progenitoras. ${ }^{5}$

$\mathrm{O}$ uso da terapia antirretroviral altamente ativa (TARV), incluindo fármacos da classe dos inibidores de protease (IP), promoveu mudanças profundas na história natural da infecção pelo HIV por induzir uma importante e sustentada supressão na replicação viral, elevando a sobrevida e a qualidade de vida dos pacientes soropositivos. ${ }^{6}$ No entanto, os benefícios associados ao uso dessa terapia antirretroviral são acompanhados por efeitos adversos, entre os quais a supressão da medula óssea e/ou anemia hemolítica evidenciada nesses pacientes. ${ }^{6,7}$

O objetivo deste estudo foi avaliar os dados hematológicos, como leucócitos, linfócitos, hematócrito, hemoglobina, contagem de células CD4+ e CD8+, carga viral e Índice de Massa Corporal (IMC), em pacientes infectados pelo HIV que faziam ou não uso de terapia antirretroviral com e sem inibidor de protease.

\section{Casuística e Métodos}

O número de pacientes ( $\mathrm{n}=250$ ) HIV soropositivos que participam de controles periódicos pelo nosso grupo vêm sendo acompanhados ao longo de cinco anos; todavia, considerando-se que, em conformidade com o consenso brasileiro, para uso de terapia antirretroviral não é preconizado o uso da terapia e que um número significativo dos pacientes em tratamento faz uso de fármacos (como reposição hormonal e outros) que potencialmente poderiam interferir nos resultados dos exames do presente estudo. Avaliamos 93 pacientes, $\mathrm{n}=45$ grupo controle (não reagente para o HIV) e $n=48$ grupo de estudo (uso de terapia antirretroviral há pelo menos seis meses com ou sem IP). A positividade para o HIV foi diagnosticada por dois métodos de ELISA e confirmados por imunofluorescência indireta, conforme recomendação do Ministério da Saúde (Brasil, 1996), com indicação clínica para iniciarem o tratamento com fármacos antirretrovirais com base nos critérios determinados pela Coordenação Nacional de Doença Sexualmente Transmissível - DST/AIDS. O grupo controle foi constituído por 45 voluntários comprovadamente soronegativos para o HIV, encaminhados pelo Banco de Sangue
Tabela 1. Características clínicas do grupo controle e dos pacientes HN soropositivos

\begin{tabular}{lcc}
\multicolumn{1}{c}{ Parâmetros } & $\begin{array}{c}\text { Grupo controle } \\
(\mathrm{n}=45)\end{array}$ & $\begin{array}{c}\mathrm{HN} \text { soropositivo } \\
(\mathrm{n}=48)\end{array}$ \\
& $38 \pm 9,2$ & $38 \pm 7,8$ \\
& & \\
& $16(35,6)$ & $22(45,8)$ \\
(n[\%]) & $29(64,4)$ & $26(54,2)$ \\
risco para o HIV (n[\%]) & & $1(2,08)$ \\
droga intravenosa & $0(0)$ & $39(81,25)$ \\
heterossexual & $45(100)$ & $7(14,58)$ \\
homossexual & $0(0)$ & $1(2,08)$ \\
bissexual & $0(0)$ & $0(0)$ \\
(n) (n[\%]) & $0(0)$ & $20(41,6)$ \\
de células T CD4+ (cels/10\%/l) & $4(8,9)$ & $0,438 \pm 0,243$ \\
de células T CD8+ (cels/10\%) & - & $1,005 \pm 0,696$ \\
do HIV (cópias/ml) & - & $18,312 \pm 51,303$ \\
\hline
\end{tabular}

do Hospital Universitário da Universidade Federal de Santa Catarina. Após a triagem clínica, os pacientes devidamente esclarecidos e que aceitaram participar da pesquisa mediante assinatura do termo de consentimento foram encaminhados ao Laboratório de Análises Clínicas do Hospital Universitário da Universidade Federal de Santa Catarina (HU/UFSC) para a coleta do material biológico (sangue). Este estudo foi aprovado pelo Comitê de Ética com Seres Humanos da Universidade Federal de Santa Catarina sob o protocolo 210/ 2005. As características clínicas dos pacientes HIV soropositivos estão sintetizadas na Tabela 1 .

\section{Parâmetros imunológicos e virulógicos}

Foram coletadas amostras de sangue para a quantificação da carga viral (RNA do HIV) por metodologia NASBA ${ }^{\circledR}$ (Nucleic Acid Sequence-Based Amplification Organon-Teknika, Boxtel, Netherlands), CD4+ e CD8+ por Citometria de Fluxo FACSCount ${ }^{\circledR}$ (Becton Dickinson, USA).

\section{Dados hematológicos}

Foram coletadas amostras de sangue com anticoagulante (EDTA) para a determinação do número de leucócitos, linfócitos, hematócrito e concentração de hemoglobina por aparelho de automação Coulter Maxim Autoloader ${ }^{\circledR}$ no setor de hematologia do Hospital Universitário de Análises Clínicas do HU/UFSC.

\section{Análise estatística}

A análise estatística univariada foi usada para avaliar a homogeneidade entre os grupos. Kolmogorov-Smirnov test foi usado para verificar se a distribuição era normal. As variáveis foram descritas como média, desvio padrão e em porcentagem. As diferenças em leucócitos, concentração de hemoglobina e IMC entre os grupos foram testadas por 
ANOVA post test Tukey. Foi considerado como índice de significância $5 \%(p<0,05)$ com intervalo de confiança de $95 \%$. Todas as análises estatísticas foram realizadas usando-se o programa estatístico SPSS (versão 13.0).

\section{Resultados}

Perfil hematológico e IMC dos grupos controle e HIV soropositivo

Os pacientes infectados pelo HIV tinham número de leucócitos significativamente menor quando comparados ao grupo controle $(\mathrm{p}<0,0001)$. Igualmente ocorreu com o IMC e a concentração de hemoglobina destes pacientes, que apresentaram IMC e concentração de hemoglobina menores quando comparados ao grupo controle $(p<0,0001$ e $p<0,006)$

Tabela 2. Perfil hematológico e IMC dos grupos controle e HIV soropositivo

\begin{tabular}{cccc}
\hline Parâmetros & $\begin{array}{c}\text { Grupo controle } \\
(\mathrm{n}=45)\end{array}$ & $\begin{array}{c}\text { HN soropositivo } \\
(\mathrm{n}=48)\end{array}$ & $p$ \\
\hline Leucócitos $\left(10^{9} / \mathrm{l}\right)$ & $6,0 \pm 1,5$ & $4,6 \pm 1,2$ & $<0,0001$ \\
Linfócitos $\left.10^{9} / \mathrm{l}\right)$ & $1,8 \pm 0,4$ & $1,6 \pm 0,8$ & 0,222 \\
Hematócrito $(\%)$ & $40,5 \pm 5,6$ & $39,3 \pm 4,8$ & 0,256 \\
Hemoglobina $(\mathrm{g} / \mathrm{dl})$ & $14,6 \pm 3,5$ & $13,0 \pm 1,6$ & 0,006 \\
IMC $\left(\mathrm{Kg} / \mathrm{m}^{2}\right)$ & $25,7 \pm 3,7$ & $22 \pm 2,8$ & $<0,0001$ \\
\hline IMC= Índice de Massa Corporal; HV= Vírus da Imunodeficiência Humana
\end{tabular}

Tabela 3. Perfil hematológico e IMC do grupo HIV soropositivo com e sem TARV

\begin{tabular}{cccc}
\hline Parâmetros & $\begin{array}{c}\text { HIV com TARV } \\
(\mathrm{n}=22)\end{array}$ & $\begin{array}{c}\text { HIV sem TARV } \\
(\mathrm{n}=26)\end{array}$ & $p$ \\
\hline Leucócitos $\left(10^{9} / \mathrm{l}\right)$ & $4,7 \pm 1,3$ & $4,6 \pm 1,0$ & 0,678 \\
Linfócitos $\left(10^{\%} /\right)$ & $1,5 \pm 0.6$ & $11,7 \pm 0,1$ & 0,530 \\
Hematócrito $(\%)$ & $39,7 \pm 5,3$ & $38,9 \pm 4,3$ & 0,568 \\
Hemoglobina $(\mathrm{g} / \mathrm{dl})$ & $13,2 \pm 1,8$ & $12,8 \pm 1,5$ & 0,467 \\
CD4+ (cels/10 $/ \mathrm{l})$ & $0.441 \pm 0,273$ & $435,4 \pm 0,218$ & 0,934 \\
CD8+ (cels/10 $/ \mathrm{l})$ & $0,928 \pm 0,441$ & $1,076 \pm 0,873$ & 0,469 \\
Carga viral (cópias/ml) & $10237,7 \pm 43708,6$ & $25739,6 \pm 57299,9$ & 0,301 \\
IMC (Kg/m $)$ & $22 \pm 2,3$ & $23,2 \pm 3,5$ & 0,712 \\
\hline
\end{tabular}

IMC= Índice de Massa Corporal; HIV= Vírus da Imunodeficiência Humana; $\mathrm{TARV}=$ Terapia antirretroviral

Tabela 3. Perfil hematológico e IMC do grupo HIV soropositivo com e sem TARV

\begin{tabular}{cccc}
\hline Parâmetros & $\begin{array}{c}\text { HIV com TARV } \\
(\mathrm{n}=22)\end{array}$ & $\begin{array}{c}\text { HIV sem TARV } \\
(\mathrm{n}=26)\end{array}$ & $p$ \\
\hline Leucócitos $\left(10^{9} / \mathrm{l}\right)$ & $4,7 \pm 1,3$ & $4,6 \pm 1,0$ & 0,678 \\
Linfócitos $\left(10^{9} / \mathrm{l}\right)$ & $1,5 \pm 0.6$ & $11,7 \pm 0,1$ & 0,530 \\
Hematócrito $(\%)$ & $39,7 \pm 5,3$ & $38,9 \pm 4,3$ & 0,568 \\
Hemoglobina $(\mathrm{g} / \mathrm{dl})$ & $13,2 \pm 1,8$ & $12,8 \pm 1,5$ & 0,467 \\
CD4+ (cels/10 $/ \mathrm{l})$ & $0.441 \pm 0,273$ & $435,4 \pm 0,218$ & 0,934 \\
CD8+ (cels/10 $/ \mathrm{l})$ & $0,928 \pm 0,441$ & $1,076 \pm 0,873$ & 0,469 \\
Carga viral $($ cópias/ml) & $10237,7 \pm 43708,6$ & $25739,6 \pm 57299,9$ & 0,301 \\
IMC $\left(\mathrm{Kg} / \mathrm{m}^{2}\right)$ & $22 \pm 2,3$ & $23,2 \pm 3,5$ & 0,712 \\
\hline
\end{tabular}

IMC= Índice de Massa Corporal; HIV= Vírus da Imunodeficiência Humana;

TARV $=$ Terapia antirretroviral respectivamente, enquanto o número de linfócitos e hematócrito não apresentaram diferença significativa entre os grupos estudados ( $\mathrm{p}=0,222 \mathrm{e} \mathrm{p}=0,256)$ respectivamente (Tabela 2).

\section{Perfil hematológico e IMC do grupo HIV soropositivo} com e sem TARV

O número de leucócitos e de linfócitos não apresentou diferença significativa entre os pacientes infectados pelo HIV que faziam tratamento com antirretroviral e aqueles que não receberam a terapia ( $p=0,678$ e $p=0,530)$ respectivamente. De forma semelhante, os pacientes HIV soropositivos submetidos ao tratamento antirretroviral com TARV não apresentaram diferença significativa nos níveis de hemoglobina, hematócrito, contagem de células T CD4+ e CD8+, carga viral e IMC, quando comparados àqueles sem uso de tratamento antirretroviral $(p=0,467$, $p=0,568, p=0,934, p=0,469, p=0,301$ e $p=0,712$ ) respectivamente (Tabela 3 ).

Perfil hematológico e IMC do grupo HIV soropositivo com uso de TARV com e sem inclusão de IP

Os pacientes HIV soropositivos submetidos ao tratamento antirretroviral foram divididos em dois grupos: os que recebiam um regime contendo IP $(\mathrm{n}=10)$ e os que recebiam um regime que não incluía IP $(\mathrm{n}=12)$. Todos os parâmetros avaliados, número de leucócitos, linfócitos, concentração de hematócrito, hemoglobina, contagem de células T CD4+, CD8+, carga viral e IMC não apresentaram diferenças significativas entre os grupos estudados ( $p=$ $0,271, p=0,133, p=0,681, \mathrm{p}=0,551, p=0,860$, $p=0,121, p=0,221$ e $p=0,960)$ respectivamente (Tabela 4).

\section{Discussão}

A prevalência de anemia em pacientes com a síndrome da imunodeficiência tem sido estimada entre $63 \%$ a $95 \% .{ }^{8}$ As causas de anemia nestes pacientes são multifatoriais, podendo ser resultado da disfunção da medula óssea pelo vírus, que, durante a infecção, provoca alterações na secreção de citocinas, imunoglobulinas e proteínas de fase aguda (APPs) como resposta do sistema imunológico da célula hospedeira. ${ }^{5,9}$ No presente estudo, puderam-se verificar diferenças significativas no número de leucócitos, concentração de hemoglobina e IMC entre os grupos controle e o HIV soropositivo (Tabela 2), sendo observada uma diminuição nestes parâmetros nos pacientes infectados pelo 
HIV. Estudos prévios demonstraram resultados semelhantes em relação ao número de leucócitos e aos níveis de hemoglobina em pacientes HIV soropositivos. Spada et al.$^{10}$ relataram diminuição de leucócitos e dos níveis de hemoglobina nestes pacientes. Os autores sugerem que o vírus HIV exerce efeito direto sobre a hematopoese. Dessa forma poderia haver comprometimento na síntese de novas células na medula óssea nos estágios iniciais da infecção, causando uma redução significativa de leucócitos e eritrócitos.

Outra hipótese seria a formação de anticorpos antieritrócitos e antileucócitos com redução na concentração de hemoglobina, provavelmente uma consequência não específica da hipergamaglobulinemia observada na infecção pelo HIV. ${ }^{11,12}$

No entanto, a hipótese mais provável para a redução dos níveis de hemoglobina é a redução dos níveis séricos de vitamina B12 nos pacientes HIV soropositivos, alterando a capacidade de ligação de cobalamina, induzida pelo HIV, que compromete a síntese das cadeias de hemoglobina. ${ }^{13}$ Esses níveis de vitamina B12 diminuídos devem-se, geralmente, pela deficiência na alimentação, infecção severa associadas a tumores no trato gastrointestinal ou diarreia, por período superior a trinta dias, os quais podem acarretar absorção insuficiente da mesma. ${ }^{4}$

A diminuição do IMC nos pacientes infectados pelo HIV, evidenciada neste estudo, pode ser explicada pela perda de peso nos mesmos, a qual é verificada com a progressão da doença. A etiologia é multifatorial, incluindo maior gasto energético pelo aumento do catabolismo e menor ingesta de nutrientes pela anorexia ou pela má absorção decorrente de infecções oportunistas. ${ }^{14,15}$

A interação entre esses fatores contribuiu para evidenciar as alterações hematológicas verificadas na infecção pelo HIV. Os efeitos da doença e o regime terapêutico podem influenciar na produção das linhagens hematopoéticas, resultando em anormalidades hematológicas. Na ausência de outros processos patológicos, a função da medula óssea já se mostra anormal na infecção pelo HIV; anemia, neutropenia e trombocitopenia são comuns com a evolução da doença. Essas alterações podem ainda ser decorrentes de infecções oportunistas que podem causar supressão da medula óssea ou induzir citopenias específicas. As terapias empregadas no tratamento da infecção também causam disfunções hematológicas por induzirem mielotoxicidade significativa. ${ }^{16}$

A TARV tem proporcionado benefícios clínicos e imunológicos aos pacientes infectados pelo HIV, ${ }^{17}$ porém, o grau de reconstituição imunológica não tem sido completamente alcançado ${ }^{18} \mathrm{e}$ tem-se verificado comprometimento clínico nos pacientes em estágio avançado da doença. ${ }^{19}$ Estudos têm demonstrado uma alta incidência de anemia em pacientes tratados com TARV. Esse efeito parece estar associado à toxicidade hematológica causada pela TARV, que pode inibir a proliferação das células sanguíneas progenitoras. ${ }^{20}$
Na avaliação do perfil hematológico dos pacientes HIV soropositivos, com e sem uso de TARV (Tabela 3), não houve diferenças significativas entre os grupos estudados quanto aos parâmetros avaliados. Berhane e colaboradores ${ }^{21}$ verificaram que o uso de TARV por um período de seis meses é suficiente para ocorrer o desenvolvimento de anemia; já o uso da mesma por um período maior pode tornar o quadro anêmico ainda mais severo. Moyle e colaboradores ${ }^{22}$ descreveram que os pacientes em uso de TARV por um período de 48 semanas desenvolviam um quadro anêmico devido à diminuição dos níveis de hemoglobina nos mesmos. Este resultado poderia ser decorrente da supressão da atividade da medula óssea induzido pelo uso de TARV

$\mathrm{Na}$ avaliação do perfil hematológico dos pacientes HIV soropositivos com um regime terapêutico com inclusão de IP e sem inclusão de IP não foram verificadas diferenças significativas entre os grupos quanto aos parâmetros avaliados (Tabela 4). A TARV é uma combinação de potentes agentes antirretrovirais, na qual pode ser incluído o IP, que tem mostrado ser eficaz na redução da carga viral no plasma e em tecidos linfoides, sustentando a supressão da replicação viral e contribuindo na reconstituição imunológica. ${ }^{23}$ No entanto, em muitos casos, essa recuperação imunológica não tem sido alcançada, podendo ser decorrente do estágio avançado da infecção, resistência medicamentosa, ou talvez o período de acompanhamento clínico da instituição da terapia tenha sido insuficiente para induzir a recuperação. ${ }^{24}$ Neste estudo, é possível que o período de instituição da terapia com IP, no regime terapêutico dos pacientes, não tenha sido suficiente para alterar os níveis dos parâmetros avaliados.

No entanto, a síndrome da imunodeficiência humana ainda está associada a anormalidades morfológicas na medula óssea e diminuição das células sanguíneas progenitoras. A depleção dessas células pode ser explicada pelo mecanismo de apoptose das mesmas, mudanças na produção de citocinas ou outros fatores imunológicos. ${ }^{25}$ Estudos têm relatado que as células progenitoras da medula óssea de pacientes infectados pelo HIV são submetidos à apoptose. E a apoptose dessas células ocorre através da Fas-L, citocina produzida por células $\mathrm{T}$ ativadas, mecanismo pelo qual as células $\mathrm{T}$ ativadas combatem as células infectadas pelo vírus $\mathrm{HIV}^{26}$

A inclusão de inibidores de protease no regime antirretroviral trouxe avanços significativos no tratamento de pacientes HIV soropositivos. A combinação de IPs com inibidores da transcriptase reversa, análogos e não análogos de nucleosídeos (NRTIs, NNRTIs) é capaz de promover a recuperação do sistema imune com o aumento sustentado da contagem de células T CD4+ e manutenção de níveis indetectáveis de carga viral do HIV. A restauração celular observada após a instituição da TARV deve-se principalmente à diminuição dos níveis de apoptose em função da diminuição da ativação imune, diminuição de proteínas do HIV que possuem ação pró-apoptótica e mesmo do efeito antiapoptótico 
de algumas drogas antirretrovirais. ${ }^{27}$ Além de exercer efeito direto na supressão da replicação do HIV, os IPs são capazes de induzir efeitos sobre as células do sistema imune. O ritonavir é capaz de modular a atividade do protossoma e do complexo maior de histocompatibilidade de classe I (MHC-I), prevenindo a apoptose e a expressão da caspase-1 em cultura de células de pacientes HIV soropositivos e de indivíduos saudáveis. ${ }^{28,29}$

\section{Considerações finais}

Os pacientes HIV soropositivos apresentaram número de leucócitos, concentração de hemoglobina e IMC menores em relação ao grupo HIV soronegativos (controle), em decorrência da redução dos parâmetros citados. Os pacientes HIV soropositivos apresentaram maior comprometimento hematológico e metabólico; assim, consequentemente, tornam-se mais suscetíveis a infecções oportunistas características da AIDS, à progressão da doença e ao alto risco de morte. Neste contexto, a avaliação dos índices hematimétricos associada à contagem de células CD4+ e quantificação da carga viral torna-se uma ferramenta importante na avaliação das condições do sistema imune, hematológico, metabólico de progressão para a AIDS, podendo auxiliar na tomada de decisões a respeito da instituição da terapia com ou sem inibidor de protease nesses pacientes.

\section{Abstract}

Anemia is the most commonly encountered hematologic abnormality in from $63 \%$ to $95 \%$ of human immunodeficiency virus (HIV)positive patients. This study intends to evaluate hematological alterations and changes in the body mass index of HIV-positive patients on antiretroviral therapy with or without protease inhibitors and those who are not on antiretroviral therapy. The HIV-positive patients were diagnosed by the ELISA test and confirmed by immunofluorescence. The hematological alterations were determined using a Coulter Maxim Autoloader ${ }^{\circledR}, C D 4+$ and $C D 8+$ cell counts by FACSCount ${ }^{\circledR}$ Flux cytometry and viral load by Nucleic Acid Sequence Based Amplification (NASBA®). The evaluation of hematological alterations showed smaller numbers of leukocytes and hemoglobin in the study group who used antiretroviral therapy compared to the control group (not on antiretroviral therapy); similar results were found for the body mass index of HIV-positive patients $(p<0.0001, p=0.006$ and $p<0.0001)$, respectively. There were no significant differences identified between the groups on antiretroviral therapy with or without protease inhibitors. An evaluation of the hematological alterations associated with CD4+ cell counts and measurement of the viral load may contribute to monitor HIV infection and assist in the decision of the most appropriate clinical intervention in these patients. Rev. Bras. Hematol. Hemoter.

Key words: Anemia; HIV/AIDS; combined modality theraphy; reverse transcriptase inhibitor.

\section{Referências Bibliográficas}

1. Edelman AS, Zolla-Pazner S. AIDS: a syndrome of immune dysregulation, dysfunction, and deficiency. FASEB J. 1989;3 (1):22-30.

2. Sullivan PS, Hanson DL, Chu SY, Jones JL, Ward JW. Epidemiology of anemia in human immunodeficiency virus (HIV)-infected persons: results from the multistate adult and adolescent spectrum of HIV disease surveillance project. Blood. 1998;91(1):301-8

3. Sullivan P. Associations of anemia, treatments for anemias, and survival in patients with human immunodeficiency virus infection. J Infect Dis. 2002;185 (Suppl 2):S138-42.

4. Volberding PA, Levine AM, Dieterich D, Mildvan D, Mitsuyasu R, Saag M. Anemia in HIV infection: clinical impact and evidencebased management strategies. Clin Infect Dis. 2004;38(10): 1454-63.

5. Claster S. Biology of anemia, differential diagnosis, and treatment options in human immunodeficiency virus infection. J Infect Dis. 2002;185 Suppl 2:S105-9.

6. Duval X, Joumont V, Leport C, Chêne G, et al. Incidence of and risk factors for adverse drug reactions in a prospective cohort of HIV-infected adults initiating protease inhibitor-containing therapy. Clin Infect Dis. 2004;39(2):248-55.

7. Morrison-Griffiths S, Newman M, O'Mahony C, Pirmohamed M. Haemolytic anaemia associated with indinavir. Postgrad Med J. 1999;75(883):313-5.

8. Groopman JE. Management of hematologic complications of human immunodeficiency virus infection. Rev Infect Dis. 1990; 12:931.

9. Jahoor F, Gazzard B, Phillips G, Sharpstone D, Delrosario M, Frazer ME, et al. The acute-phase protein response to human immunodeficiency virus infection in human subjects. Am J Physiol. 1999;276(6 Pt 1):E1092-8.

10. Spada C, Treintinger A, Hoshikawa-Fujimura AY. HIV influence on hemapoiesis at he initial stage of infections. Eur J Haematol. 1998;58:1-5

11. van der Lelie J, Lange JM, Vos JJ, van Dalen CM, Danner SA, von dem Borne AE. Autoimmunity against blood cells in human immunodeficiency virus (HIV) infection. Br J Haematol. 1987;67 (1):109-14

12. Riera NE, Galassi N, Barrera S, Rickard E, Muchimik G, PerezBianco R, et al. Anti-leukocyte antibodies as a consequence of HIV infection in HIV+ individuals. Immunol Lett. 1992;33 (1):99-104.

13. Burkes RL, Cohen H, Krailo M, Sinow RM, Carmel R. Low serum cobalamin levels occur frequently in the acquired immunedeficiency syndrome and related disorders. Eur J Haematol. 1987; 38(2):141-7.

14. Luder E, Godfrey E, Godbold J, Simpson DM. Assessment of nutritional, clinical, and immunologic status of HIV-infected, innercity patients with multiple risk factors. J Am Diet Assoc. 1995; 95(6):655-60.

15. Jiménez-Expósito MJ, García-Lorda P, Alonso-Villaverde C, de Vírgala CM, Solà R, Masana L, et al. Effect of malabsorption on nutritional status and resting energy expenditure in HIV-infected patients. AIDS. 1998;12(15):1965-72.

16. Evans RH, Scadden DT. Haematological aspects of HIV infection. Baillieres Best Pract Res Clin Haematol. 2000;13(2):215-30.

17. Bart PA, Rizzardi GP, Tambussi G, et al. Immunological and virological responses in HIV-1-infected adults at early stage of established infection treated with highly active antiretroviral therapy. AIDS. 2000;14:1887-97. 
18. Roger RM, Breittmayer JP, Arlotto C, et al. Highly active antiretroviral therapy (HAART) is associated with a lower level of CD4 + T cell apoptosis in HIV-infected patients. Clin. Exp. Immunol. 1999;188:412-6.

19. Lederman MM, Connick E, Landay A, et al. Immunolgic responses associated with 12 weeks of combination antiretroviral therapy consisting of zidovudine, lamivudine and ritonavir: results of AIDS Clinical Trials Group Protocol 315. J Infect Dis. 1998; 178(1):70-9.

20. Curkendall SM, Richardson JT, Emons MF, Fisher AE, Everhard F. Incidence of anaemia among HIV-infected patients treated with highly active antiretroviral therapy. HIV Med. 2007;8(8): 483-90.

21. Berhane K, Karim R, Chohen MH, Masri-Lavine L, Young M, Anastos $\mathrm{K}$, et al. Impact of highly active antiretroviral therapy on anemia and relationship between anemia and survival in a larg cohort of HIV-infected women. J Acquir Immune Defic Syndr. 2004;37(2):1245-52.

22. Moyle G, Sawyer W, Law M, Amin J, Hill A. Changes in hematologic parameters and efficacy os thymidine analogue-based, highly active antiretroviral therapy: A meta-analysis of six prospective, randomized, comparative studies. Clin Ther. 2004;26(1):92-7.

23. Perelson AS, Essunger P, Cao Y, Vesanen M, Hurley A, Saksela K, et al. Decay characteristics of HIV-1-infected compartments during combination therapy. Nature 1997;387(6629):188-91.

24. Plana M, García F, Gallart T, Tortajada C, Soriano A, Palou E, et al. Immunological benefits of antiretroviral therapy in very early stages of asymptomatic chronic HIV-1 infection. AIDS. 2000;14 (13):1921-33.

25. Kobari L, Giarratana MC, Poloni A, et al. Flt 3 ligand, MGDF, Epo and G-CSF enhance ex vivo expansion of hematopoietic cell compartments in the presence of SCF, IL-3 and IL-6. Bone Marrow Transplant. 1998;21(8):759-67.

26. Zauli G, Capitani S. HIV-1-related mechanisms of suppression of CD34 + hematopoietic progenitors. Pathobiology. 1996; 64 (1):53-8

27. Gougeon ML. Apoptosis as an HIV strategy to escape immune attack. Nat Rev Immunol. 2003;3(5):392-404.

28. Sloand EM, Maciejewski J, Kumar P, Kim S, Chaudhuri A, Young N. Protease inhibitor stimulate hematopoiesis and decrease apoptosis and ICE expression in CD34 (+) cells. Blood. 2000;96 (8):2735-9

29. Baggio GL, Spada C. Contribuição da apoptose na diminuição da contagem de células T CD4 no paciente HIV. Tendências em HIVAIDS. 2008;3(1):18-22.

Avaliação: Editor e dois revisores externos

Conflito de interesse: sem conflito de interesse

Recebido: 21/08/2008

Aceito após modificações: 13/10/2009 